\title{
The Hilbert-Schmidt Analyticity Associated with Infinite-Dimensional Unitary Groups
}

\author{
Oleh Lopushansky
}

\begin{abstract}
The article is devoted to the problem of Hilbert-Schmidt type analytic extensions in Hardy spaces over the infinite-dimensional unitary group endowed with an invariant probability measure. Reproducing kernels of Hardy spaces, integral formulas of analytic extensions and their boundary values are considered.
\end{abstract}

Mathematics Subject Classification. Primary 46T12; Secondary 46G20.

Keywords. Measures on infinite-dimensional manifolds, Hardy spaces on infinite-dimensional domains, Hilbert-Schmidt analyticity.

\section{Introduction}

The paper deals with the problem of Hilbert-Schmidt type analytic extensions in the Hardy space $H_{\chi}^{2}$ of complex functions over the infinite-dimensional group $U(\infty)=\bigcup\{U(m): m \in \mathbb{N}\}$ endowed with an invariant probability measure $\chi$ where $U(m)$ are subgroups of unitary $m \times m$-matrices. The measure $\chi$ is defined as a projective limit $\chi=\lim \chi_{m}$ of the Haar probability measures $\chi_{m}$ on $U(m)$. Moreover, $\chi$ is supported by a projective limit $\mathfrak{U}=\lim U(m)$ and is invariant under the right action of $U^{2}(\infty):=U(\infty) \times U(\infty)$ on $\mathfrak{U}$.

A goal of this work is to find integral formulas for Hilbert-Schmidt analytic extensions of functions from $H_{\chi}^{2}$ and to describe their radial boundary values on the open unit ball in a Hilbert space $\mathrm{E}$ where $U(\infty)$ acts irreducibly.

The measure $\chi$ on $\mathfrak{U}$ was described by Olshanski [13] and Neretin [12]. The notion $\mathfrak{U}$ is related to Pickrell's space of a virtual Grassmannian [16]. Hardy spaces in infinite-dimensional settings were discussed in the works of Cole and Gamelin [5], Ørsted and Neeb [14]. Spaces of analytic functions of HilbertSchmidt holomorphy types were considered by Dwyer III [6] and Petersson [15]. 
More general classes of analytic functions associated with coherent sequences of polynomial ideals were described by Carando et al. [4]. Integral formulas for analytic functions employing Wiener measures on infinite-dimensional Banach spaces were suggested by Pinasco and Zalduendo [17].

Note that spaces of integrable functions with respect to invariant measures over infinite-dimensional groups have been widely applied in stochastic processes $[2,3]$, as well as in other areas.

This paper presents the following results. In Theorem 3.2, we describe an orthogonal basis in the Hardy space $H_{\chi}^{2}$ indexed by means of Yang diagrams, consisting of $\chi$-essentially bounded functions. Using this basis, in Theorem 4.2 the reproducing kernel of $H_{\chi}^{2}$ is calculated. It also allows us to define an antilinear isometric isomorphism $\mathcal{J}$ between $H_{\chi}^{2}$ and the symmetric Fock space $\Gamma$ generated by $\mathrm{E}$. This isomorphism equips $H_{\chi}^{2}$ with a suitable infinitedimensional analytic structure. By means of $\mathcal{J}$, we establish in Theorem 6.2 an integral formula for Hilbert-Schmidt analytic extensions of functions from $H_{\chi}^{2}$ on the open unit ball $\mathrm{B} \subset \mathrm{E}$. The radial boundary values of these analytic extensions are described in Theorem 7.1.

\section{Background on Invariant Measure}

Let $U(m)(m \in \mathbb{N})$ be the group of unitary $(m \times m)$-matrices. We endow $U(\infty)=\bigcup U(m)$ with the inductive topology under every continuous inclusion $U(m) \uparrow U(\infty)$ which assigns to any $u_{m} \in U(m)$ the matrix $\left[\begin{array}{cc}u_{m} & 0 \\ 0 & \mathbb{1}\end{array}\right] \in U(\infty)$. The right action over $U(\infty)$ is defined via

$$
u . g=w^{-1} u v, \quad u \in U(\infty), \quad g=(v, w) \in U^{2}(\infty)
$$

(the right action over $U(m)$ is defined similarly with $u \in U(m)$ and $g=$ $(v, w) \in U^{2}(m)$ where $\left.U^{2}(m):=U(m) \times U(m)\right)$.

Following [12,13], every $u_{m} \in U(m)$ with $m>1$ can be written as $u_{m}=$ $\left[\begin{array}{cr}z_{m-1} & a \\ b & t\end{array}\right]$ so that $z_{m-1}$ is a $(m-1) \times(m-1)$-matrix and $t \in \mathbb{C}$. It was proven that the Livšic-type mapping (which is not a group homomorphism)

$$
\pi_{m-1}^{m}: u_{m} \longmapsto u_{m-1}:=\left\{\begin{array}{lr}
z_{m-1}-\left[a(1+t)^{-1} b\right]: & t \neq-1 \\
z_{m-1}: & t=-1
\end{array}\right.
$$

from $U(m)$ onto $U(m-1)$ is Borel and surjective.

Consider the projective limit $\mathfrak{U}=\lim U(m)$ taken with respect to $\pi_{m-1}^{m}$. The embedding $\rho: U(\infty) \leftrightarrow \mathfrak{U}$ assigns to every $u_{m} \in U(m)$ the stabilized sequence $u=\left(u_{k}\right)_{k \in \mathbb{N}}($ see $[13$, n.4]) so that 


$$
\rho: U(m) \ni u_{m} \longmapsto\left(u_{k}\right) \in \mathfrak{U}, \quad u_{k}=\left\{\begin{array}{c}
\pi_{k}^{m}\left(u_{m}\right): k<m, \\
u_{m}: k=m, \\
{\left[\begin{array}{cc}
u_{m} & 0 \\
0 & \mathbb{1}
\end{array}\right]: k>m}
\end{array}\right.
$$

where the projections $\pi_{m}: \mathfrak{U} \ni u \longrightarrow u_{m} \in U(m)$ such that $\pi_{m-1}^{m} \circ \pi_{m}=\pi_{m-1}$ are surjective and $\pi_{k}^{m}:=\pi_{k}^{k+1} \circ \cdots \circ \pi_{m-1}^{m}$ for $k<m$. Using (2.1), the right action of $U^{2}(\infty)$ over $\mathfrak{U}$ can be defined as

$$
\pi_{m}(u . g)=w^{-1} \pi_{m}(u) v, \quad u \in \mathfrak{U}
$$

where $m$ is so large that $g=(v, w) \in U^{2}(m)$ (see [13, Def 4.5]).

We endow every group $U(m)$ with the probability Haar measure $\chi_{m}$. It is known [12, Thm 1.6] that the pushforward of $\chi_{m}$ to $U(m-1)$ under $\pi_{m-1}^{m}$ is the probability Haar measure $\chi_{m-1}$ on $U(m)$. Let $U^{\prime}(m)$ be the subset in $U(m)$ of matrices which do not have -1 as an eigenvalue. Then $U^{\prime}(m)$ is open in $U(m)$ and $U(m) \backslash U^{\prime}(m)$ is $\chi_{m}$-negligible. Moreover, the restriction $\pi_{m-1}^{m}: U^{\prime}(m) \longrightarrow U^{\prime}(m-1)$ is continuous and surjective [13, Lem. 3.11].

Following [13, Lem. 4.8], [12, n.3.1], via of the Kolmogorov consistency theorem we uniquely define on $\mathfrak{U}$ the probability measure $\chi$ which is the projective limit under the mapping (2.2), i.e., we put

$$
\chi=\lim _{\longleftarrow} \chi_{m} \text { with } \quad \chi_{m}=\chi \circ \pi_{m}^{-1} \text { for all } m \in \mathbb{N} .
$$

If $\mathfrak{U}^{\prime}=\lim _{\longleftarrow} U^{\prime}(m)$ is the projective limit with respect to $\left.\pi_{m-1}^{m}\right|_{U^{\prime}(m)}$ then $\mathfrak{U} \backslash \mathfrak{U}^{\prime}$ is $\chi$-negligible, because $\chi_{m}$ is zero on $U(m) \backslash U^{\prime}(m)$ for any $m$.

A complex-valued function on $\mathfrak{U}$ is called cylindrical if it has the form $f=f_{m} \circ \pi_{m}$ for a certain $m \in \mathbb{N}$ and a complex function $f_{m}$ on $U(m)[13$, Def. 4.5]. By $L_{\chi}^{\infty}$ we denote the closed linear hull of all cylindrical $\chi$-essentially bounded Borel functions endowed with the norm $\|f\|_{L_{\chi}^{\infty}}=\operatorname{ess}_{\sup _{u \in \mathfrak{U}}}|f(u)|$.

The measure (2.5) is a probability measure and is $U^{2}(\infty)$-invariant under the right actions (2.4) over $\mathfrak{U}$ [12, Prop. 3.2]. Moreover, this measure is Radon so that

$$
\int_{\mathfrak{U}} f(u . g) d \chi(u)=\int_{\mathfrak{U}} f(u) d \chi(u), \quad g \in U^{2}(\infty), \quad f \in L_{\chi}^{\infty}
$$

and it satisfies the property: $\left(\chi \circ \pi_{m}^{-1}\right)(K)=\chi_{m}(K)$ for any compact set $K$ in $U(m)[11$, Lem. 1]. Using the invariance property (2.6) and the Fubini theorem (see [11, Lem. 2]), we obtain

$$
\begin{aligned}
& \int_{\mathfrak{U}} f d \chi=\int_{\mathfrak{U}} d \chi(u) \int_{U^{2}(m)} f(u \cdot g) d\left(\chi_{m} \otimes \chi_{m}\right)(g), \\
& \int_{\mathfrak{U}} f d \chi=\frac{1}{2 \pi} \int_{\mathfrak{U}} d \chi(u) \int_{-\pi}^{\pi} f[\exp (\dot{\mathrm{i}} \vartheta) u] d \vartheta
\end{aligned}
$$


for all $f \in L_{\chi}^{\infty}$. The closed linear hull of cylindrical complex functions endowed with the norm $\|f\|_{L_{\chi}^{2}}=\left(\int_{\mathfrak{U}}|f|^{2} d \chi\right)^{1 / 2}$ is denoted by $L_{\chi}^{2}$. It is clear that $L_{\chi}^{\infty} \leftrightarrow L_{\chi}^{2}$ and $\|f\|_{L_{\chi}^{2}} \leq\|f\|_{L_{\chi}^{\infty}}$ for all $f \in L_{\chi}^{\infty}$.

\section{Hardy Spaces}

Throughout the paper $\mathrm{E}$ is a separable complex Hilbert space with an orthonormal basis $\left\{\mathfrak{e}_{k}: k \in \mathbb{N}\right\}$, scalar product $\langle\cdot \mid \cdot\rangle$ and norm $\|\cdot\|=\langle\cdot \mid \cdot\rangle^{1 / 2}$. So, for any element $x \in \mathrm{E}$ the following Fourier decomposition holds,

$$
x=\sum \mathfrak{e}_{k} \hat{x}_{k}, \quad \hat{x}_{k}=\left\langle x \mid \mathfrak{e}_{k}\right\rangle .
$$

In what follows, let $\mathrm{B}=\{x \in \mathrm{E}:\|x\|<1\}$ and $\mathrm{S}=\{x \in \mathrm{E}:\|x\|=1\}$.

Let $\mathrm{E}^{\otimes n}$ be the complete $n$th tensor power of $\mathrm{E}$ endowed with the scalar product and norm

$$
\langle\psi \mid \phi\rangle=\left\langle x_{1} \mid y_{1}\right\rangle \cdots\left\langle x_{n} \mid y_{n}\right\rangle, \quad\|\psi\|=\langle\psi \mid \psi\rangle^{1 / 2}
$$

for all $\psi=x_{1} \otimes \cdots \otimes x_{n}, \phi=y_{1} \otimes \cdots \otimes y_{n} \in \mathrm{E}^{\otimes n}$ with $x_{i}, y_{i} \in \mathrm{E}(i=1, \ldots, n)$. As $\sigma:\{1, \ldots, n\} \longmapsto\{\sigma(1), \ldots, \sigma(n)\}$ runs through all $n$-elements permutations, the symmetric complete $n$th tensor power $\mathrm{E}^{\odot} n$ is defined to be a codomain of the orthogonal projector

$$
\mathrm{E}^{\otimes n} \ni \psi \longmapsto x_{1} \odot \cdots \odot x_{n}:=\frac{1}{n !} \sum_{\sigma} x_{\sigma(1)} \otimes \cdots \otimes x_{\sigma(n)} \in \mathrm{E}^{\odot n} .
$$

Note that $x^{\otimes n}=x \otimes \cdots \otimes x=x \odot \cdots \odot x=x^{\odot n}$. Put $\mathrm{E}^{\otimes 0}=\mathrm{E}^{\odot 0}=\mathbb{C}$.

Let $\lambda=\left(\lambda_{1}, \ldots, \lambda_{m}\right) \in \mathbb{N}^{m}$ be a partition of an integer $n \in \mathbb{N}$ with $m \leq n$ and $\lambda_{1} \geq \lambda_{2} \geq \cdots \lambda_{m}>0$, i.e., $|\lambda|=n$ where $|\lambda|:=\lambda_{1}+\cdots+\lambda_{m}$. We identify partitions with Young diagrams. By $\ell(\lambda)=m$ we denote the length of $\lambda$ defined as the number of rows in $\lambda$. Let $\mathbb{Y}$ denote all Young diagrams and $\mathbb{Y}_{n}:=\{\lambda \in \mathbb{Y}:|\lambda|=n\}$. Assume that $\mathbb{Y}$ includes the empty partition $\emptyset=(0,0, \ldots)$.

An orthogonal basis in $\mathrm{E}^{\odot n}$ is formed by the system of symmetric tensor products (see e.g. [1, Sec. 2.2.2])

$$
\mathfrak{e}^{\odot \mathbb{Y}_{n}}=\bigcup_{\lambda \in \mathbb{Y}_{n}}\left\{\mathfrak{e}_{\imath}^{\odot \lambda}:=\mathfrak{e}_{\imath_{1}}^{\otimes \lambda_{1}} \odot \cdots \odot \mathfrak{e}_{\imath_{m}}^{\otimes \lambda_{m}}: \imath \in \mathbb{N}_{*}^{m}, m=\ell(\lambda)\right\}, \quad \mathfrak{e}_{\imath}^{\odot \emptyset}=1
$$

where $\mathbb{N}_{*}^{m}:=\left\{\imath=\left(\imath_{1}, \ldots, \imath_{m}\right) \in \mathbb{N}^{m}: \imath_{j} \neq \imath_{k}, \forall j \neq k\right\}$. As is well known,

$$
\left\|\mathfrak{e}_{\imath}^{\odot \lambda}\right\|^{2}=\frac{\lambda !}{|\lambda| !}, \quad \lambda !:=\lambda_{1} ! \cdots \lambda_{m} !
$$

In what follows, we will use the fact that for every $\psi \in \mathrm{E}^{\odot n}$ one can uniquely define the so-called Hilbert-Schmidt $n$-homogenous polynomial

$$
\psi^{*}(x):=\left\langle x^{\otimes n} \mid \psi\right\rangle, \quad x \in \mathrm{E} .
$$


In fact, the polarization formula for symmetric tensor products (see $[8,1.5]$ )

$$
z_{1} \odot \cdots \odot z_{n}=\frac{1}{2^{n} n !} \sum_{\theta_{1}, \ldots, \theta_{n}= \pm 1} \theta_{1} \ldots \theta_{n} x^{\otimes n}, \quad x=\sum_{k=1}^{n} \theta_{k} z_{k}
$$

$\left(z_{1}, \ldots, z_{n} \in \mathrm{E}\right)$ implies that the $n$-homogenous polynomial $\left\langle x^{\otimes n} \mid \psi\right\rangle$ is uniquely determines $\psi$, because the set of all $z_{1} \odot \cdots \odot z_{n}$ is total in $\mathrm{E}^{\odot n}$.

Using the embedding (2.3), we define the E-valued mapping

$$
\zeta: \mathfrak{U} \ni u \longmapsto \rho^{-1}(u) \mathfrak{e}_{1}
$$

which do not depend on the choice of $\mathfrak{e}_{1}$ in

$$
\mathrm{S}(\infty):=\{\zeta(u): u \in \mathfrak{U}\}=\bigcup\{\mathrm{S}(m): m \in \mathbb{N}\}
$$

where $\mathbf{S}(m)$ is the $m$-dimensional unit sphere. In fact, for each stabilized sequence $u=\left(u_{k}\right) \in \mathfrak{U}$ there exists an index $m$ such that $\rho^{-1}(u) \mathfrak{e}_{1}=u_{k} \mathfrak{e}_{1}$ belongs to $\mathrm{S}(m)$ for all $k \geq m$. On the other hand, for each $\mathfrak{e} \in \mathbf{S}(k)$ there exists $v \in U(k)$ such that $v \mathfrak{e}=\mathfrak{e}_{1}$. Defining $u . g \in \mathfrak{U}$ with $g=(1, v) \in U^{2}(k)$ by means of $(2.3)-(2.4)$, we have $\rho^{-1}(u . g) \mathfrak{e}=\pi_{k}(u . g) \mathfrak{e}=\pi_{k}(u) \mathfrak{e}_{1}=\rho^{-1}(u) \mathfrak{e}_{1}$.

Consider the following system of cylindrical Borel functions

$$
\varepsilon_{k}(u):=\left\langle\zeta(u) \mid \mathfrak{e}_{k}\right\rangle, \quad k \in \mathbb{N}
$$

where $\varepsilon_{k}:=\mathfrak{e}_{k}^{*} \circ \zeta$. Using $\zeta$, we may define the $\mathrm{E}^{\odot n}$-valued Borel mapping

$$
\zeta^{\otimes n}: \mathfrak{U} \ni u \longmapsto \underbrace{\zeta(u) \otimes \cdots \otimes \zeta(u)}, \quad \zeta^{\otimes 0} \equiv 1 .
$$

The following assertion, which is a consequence of the polarization formula (3.3), is proved in [11, Lem. 3].

Lemma 3.1. The equality $\mathrm{S}(\infty)=\left\{\zeta(u): u \in \mathfrak{U}^{\prime}\right\}$ holds. As a consequence, to every $\psi \in \mathrm{E}_{\imath}^{\odot n}$ there uniquely corresponds the function in $L_{\chi}^{\infty}$

$$
\psi_{\zeta}(u):=\left\langle\zeta^{\otimes n}(u) \mid \psi\right\rangle, \quad u \in \mathfrak{U}
$$

given by continuous restriction to $\mathfrak{U}^{\prime}$. In particular, to every $\mathfrak{e}_{i}^{\odot \lambda} \in \mathfrak{e}^{\odot \mathbb{Y}_{n}}$ there corresponds in $L_{\chi}^{\infty}$ the cylindrical function in the variable $u \in \mathfrak{U}$,

$$
\varepsilon_{\imath}^{\lambda}(u):=\left\langle\zeta^{\otimes n}(u) \mid \mathfrak{e}_{\imath}^{\odot \lambda}\right\rangle=\prod_{k=1}^{\ell(\lambda)}\left\langle\zeta(u) \mid \mathfrak{e}_{\imath_{k}}\right\rangle^{\lambda_{k}} .
$$

Lemma 3.1 straightforwardly implies that the system $\mathfrak{e}^{\odot \mathbb{Y}}:=\bigcup \mathfrak{e}^{\odot \mathbb{Y}_{n}}$ of tensor products $\mathfrak{e}_{\imath}^{\odot \lambda}=\mathfrak{e}_{\imath_{1}}^{\otimes \lambda_{1}} \odot \cdots \odot \mathfrak{e}_{\imath_{m}}^{\otimes \lambda_{m}}$, indexed by $\lambda=\left(\lambda_{1}, \ldots, \lambda_{m}\right) \in \mathbb{Y}$ and $\imath=\left(\imath_{1}, \ldots, \imath_{m}\right) \in \mathbb{N}_{*}^{m}$ with $m=\ell(\lambda)$, uniquely defines the appropriate system

$$
\varepsilon^{\mathbb{Y}}:=\bigcup_{\lambda \in \mathbb{Y}}\left\{\varepsilon_{\imath}^{\lambda}:=\varepsilon_{\imath_{1}}^{\lambda_{1}} \cdots \cdot \varepsilon_{\imath_{m}}^{\lambda_{m}}: \imath \in \mathbb{N}_{*}^{m}, m=\ell(\lambda)\right\}, \quad \varepsilon_{\imath}^{\emptyset} \equiv 1,
$$

of $\chi$-essentially bounded cylindrical functions in the variable $u \in \mathfrak{U}$ that possess continuous restrictions to $\mathfrak{U}^{\prime}$. 
Theorem 3.2. For any $\imath \in \mathbb{N}_{*}^{m}$ and $\psi, \phi \in \mathrm{E}_{\imath}^{\odot n}$, the following equality holds,

$$
\left(\begin{array}{c}
n+m-1 \\
n
\end{array}\right) \int_{\mathfrak{U}} \phi_{\zeta} \bar{\psi}_{\zeta} d \chi=\langle\psi \mid \phi\rangle .
$$

As a consequence, given $(\lambda, \imath) \in \mathbb{Y} \times \mathbb{N}_{*}^{m}$ with $m=\ell(\lambda)$, the system $\varepsilon^{\mathbb{Y}}$ of functions $\varepsilon_{\imath}^{\lambda}$ is orthogonal in the space $L_{\chi}^{2}$ and

$$
\left\|\varepsilon_{\imath}^{\lambda}\right\|_{L_{\chi}^{2}}=\left(\frac{(m-1) ! \lambda !}{(m-1+|\lambda|) !}\right)^{1 / 2} .
$$

Proof. Let $\mathrm{E}_{\imath}$ with $\imath=\left(\imath_{1}, \ldots, \imath_{m}\right) \in \mathbb{N}_{*}^{m}$ be the $m$-dimensional subspace in $\mathrm{E}$ spanned by $\left\{\mathfrak{e}_{\imath_{1}}, \ldots, \mathfrak{e}_{\imath_{m}}\right\}$ and $U(\imath)$ be the unitary subgroup of $U(\infty)$ acting in $\mathrm{E}_{\imath}$. The symbol $\mathrm{E}_{\imath}^{\odot n}$ means the $n$th symmetric tensor power of $\mathrm{E}_{\imath}$. Briefly denote $\psi_{\dagger}[v \zeta(u)]:=\left\langle\left(\left[v \rho^{-1}(u)\right] \mathfrak{e}_{1}\right)^{\otimes n} \mid \psi\right\rangle$ with $\psi \in \mathrm{E}_{\imath}^{\odot n}$ for all $v \in U(\imath)$ and $u \in \mathfrak{U}$. Using (2.7) with $U(\imath)$ instead of $U(m)$, we have

$$
\int_{\mathfrak{U}} \phi_{\zeta} \bar{\psi}_{\zeta} d \chi=\int_{\mathfrak{U}} d \chi(u) \int_{U(\imath)} \phi_{\dagger}[v \zeta(u)] \cdot \bar{\psi}_{\dagger}[v \zeta(u)] d \chi_{\imath}(v)
$$

for all $\psi, \phi \in \mathrm{E}_{\imath}^{\odot n}$. It is clear that

$$
\left|\int_{U(\imath)} \phi_{\dagger} \bar{\psi}_{\dagger} d \chi_{\imath}\right| \leq \sup _{v \in U(\imath)}\left|\phi_{\dagger}[v \zeta(u)]\right|\left|\psi_{\dagger}[v \zeta(u)]\right\rangle \mid \leq\|\phi\|\|\psi\|
$$

for all $u \in \mathfrak{U}$. Hence, the corresponding sesquilinear form in (3.7) is continuous on $\mathrm{E}_{\imath}^{\odot n}$. Thus, there exists a linear bounded operator $A$ over $\mathrm{E}_{\imath}^{\odot n}$ such that

$$
\langle A \psi \mid \phi\rangle=\int_{U(\imath)} \phi_{\dagger} \bar{\psi}_{\dagger} d \chi_{\imath} .
$$

Next we show that $A$ commutes with all operators $w^{\otimes n} \in \mathscr{L}\left(\mathrm{E}_{\imath}^{\odot n}\right)$ with $w \in U(\imath)$ acting as $w^{\otimes n} x^{\otimes n}=(w x)^{\otimes n},\left(x \in \mathrm{E}_{\imath}\right)$. Invariance properties (2.6) of $\chi_{\imath}$ under the right action (2.4) yield

$$
\begin{aligned}
& \left\langle\left(A \circ w^{\otimes n}\right) \psi \mid \phi\right\rangle \\
& =\int_{U(\imath)}\left\langle[v \zeta(u)]^{\otimes n} \mid \phi\right\rangle \overline{\left\langle[v \zeta(u)]^{\otimes n} \mid w^{\otimes n} \psi\right\rangle} d \chi_{\imath}(v) \\
& =\int_{U(\imath)}\left\langle\left[w^{-1} v \zeta(u)\right]^{\otimes n} \mid\left(w^{-1}\right)^{\otimes n} \phi\right\rangle \overline{\left\langle\left[w^{-1} v \zeta(u)\right]^{\otimes n} \mid \psi\right\rangle} d \chi_{\imath}(v) \\
& =\int_{U(\imath)}\left\langle[v \zeta(u)]^{\otimes n} \mid\left(w^{-1}\right)^{\otimes n} \phi\right\rangle \overline{\left\langle[v \zeta(u)]^{\otimes n} \mid \psi\right\rangle} d \chi_{\imath}(v) \\
& =\left\langle A \psi \mid\left(w^{-1}\right)^{\otimes n} \phi\right\rangle=\left\langle\left(w^{\otimes n} \circ A\right) \psi \mid \phi\right\rangle,
\end{aligned}
$$

where $w^{-1} \in U(\imath)$ is the hermitian adjoint matrix of $w$. Hence, the equality

$$
A \circ w^{\otimes n}=w^{\otimes n} \circ A, \quad w \in U(\imath)
$$


holds. Let us check that the operator $A$, satisfying the condition (3.8), is proportional to the identity operator on $\mathrm{E}_{\imath}^{\otimes n}$. To this end we form the $n$th tensor power of the unitary group $U(\imath)$,

$$
[U(\imath)]^{\otimes n}=\left\{w^{\otimes n} \in \mathscr{L}\left(\mathrm{E}_{\imath}^{\odot n}\right): w \in U(\imath)\right\}, \quad[U(\imath)]^{\otimes 0}=1 .
$$

Clearly, $[U(\imath)]^{\otimes n}$ is a unitary group over $\mathrm{E}_{\imath}^{\odot n}$. Let us check that the corresponding unitary representation

$$
U(\imath) \ni w \longmapsto w^{\otimes n} \in \mathscr{L}\left(\mathrm{E}_{\imath}^{\odot n}\right)
$$

is irreducible. This means that there is no subspace in $\mathrm{E}_{\imath}^{\odot} n$ other than $\{0\}$ and the whole space which is invariant under the action of $[U(\imath)]^{\otimes n}$.

Suppose, on the contrary, that there is an element $\psi \in \mathrm{E}_{\imath}^{\odot} n$ such that the equality $\left\langle\left(\left[w \rho^{-1}(u)\right] \mathfrak{e}_{1}\right)^{\otimes n} \mid \psi\right\rangle=0$ holds for all $w \in U(\imath)$ and $u \in U(\infty)$. By Lemma 3.1 the elements $w \rho^{-1}(u)$ act transitively on $\mathrm{S}(\infty)$. Hence, by $n$ homogeneity, we obtain $\left\langle x^{\otimes n} \mid \psi\right\rangle=0$ for all $x \in \mathrm{E}_{\imath}$. Applying the polarization formula (3.3), we get $\psi=0$. Hence, (3.9) is irreducible.

Thus, we can apply to (3.9) the Schur lemma [10, Thm 21.30]: a nonzero matrix which commutes with all matrices of an irreducible representation is a constant multiple of the unit matrix. As a result, we obtain that the operator $A$, satisfying $(3.8)$, is proportional to the identity operator on $\mathrm{E}_{\imath}^{\odot n}$ i.e. $A=\alpha_{(n, \imath)} \mathbb{1}_{\mathrm{E}_{\imath}^{\odot n}}$ with a constant $\alpha_{(n, \imath)}>0$. It follows that

$$
\int_{U(\imath)} \phi_{\dagger} \bar{\psi}_{\dagger} d \chi_{\imath}=\alpha_{(n, \imath)}\langle\psi \mid \phi\rangle, \quad \phi, \psi \in \mathrm{E}_{\imath}^{\odot n} .
$$

In particular, the subsystem of cylindrical functions $\varepsilon_{\imath}^{\lambda}$ with a fixed $\imath \in \mathbb{N}_{*}^{m}$ is orthogonal in $L_{\chi}^{2}$, because the corresponding system of tensor products $\mathfrak{e}_{\imath}^{\odot \lambda}$ indexed by $\lambda \in \mathbb{Y}_{n}$ with $\ell(\lambda)=m$ forms an orthogonal basis in $\mathrm{E}_{\imath}^{\odot n}$.

It remains to note that the set of all indices $\imath=\left(\imath_{1}, \ldots, \imath_{m}\right) \in \mathbb{N}_{*}^{m}$ with all $m=\ell(\lambda)$ is directed with respect to the set-theoretic embedding, i.e., for any $\imath, \imath^{\prime}$ there exists $\imath^{\prime \prime}$ so that $\imath \cup \imath^{\prime} \subset \imath^{\prime \prime}$. This fact and the above reasoning imply that the whole system $\varepsilon^{\mathbb{Y}}$ is also orthogonal in $L_{\chi}^{2}$.

Taking into account (3.2), we can choose $\phi_{n}=\psi_{n}=\varepsilon_{\imath}^{\lambda} \sqrt{n ! / \lambda !}$ in (3.10). As a result, we obtain

$$
\alpha_{(n, \imath)}=\frac{n !}{\lambda !} \int_{U(\imath)}\left|\varepsilon_{\imath}^{\lambda}\right|^{2} d \chi_{\imath}=\frac{n !}{\lambda !}\left\|\varepsilon_{\imath}^{\lambda}\right\|_{L_{\chi}^{2}}^{2} .
$$

The well known formula $[18,1.4 .9]$ for the unitary $m$-dimensional group gives

$$
\int_{U(\imath)}\left|\varepsilon_{\imath}^{\lambda}\right|^{2} d \chi_{\imath}=\frac{\lambda !(m-1) !}{(n+m-1) !}, \quad|\lambda|=n, \quad \ell(\lambda)=m .
$$

Using the last two formulas, we arrive at the relation

$$
\alpha_{(n, \imath)}=\frac{n !}{\lambda !} \int_{U(\imath)}\left|\varepsilon_{\imath}^{\lambda}\right|^{2} d \chi_{\imath}=\frac{n !}{\lambda !} \frac{\lambda !(m-1) !}{(n+m-1) !}=\frac{n !(m-1) !}{(n+m-1) !} .
$$


Combining (3.7) and (3.11), we get (3.5) and, as a consequence, (3.6).

Definition 3.3. By $H_{\chi}^{2}$ we denote the Hardy space over $U(\infty)$ defined as the $L_{\chi}^{2}$-closure of the complex linear span of the orthogonal system $\varepsilon^{\mathbb{Y}}$.

Let the space $H_{\chi}^{2, n}$ be the $L_{\chi}^{2}$-closure of the complex linear span of the subsystem $\varepsilon^{\mathbb{Y}_{n}}:=\left\{\varepsilon_{\imath}^{\lambda} \in \varepsilon^{\mathbb{Y}}:(\lambda, \imath) \in \mathbb{Y}_{n} \times \mathbb{N}_{*}^{\ell(\lambda)}\right\}$ with a fixed $n \in \mathbb{Z}_{+}$.

Corollary 3.4. For any positive integers $n \neq k$ the orthogonality $H_{\chi}^{2, n} \perp H_{\chi}^{2, k}$ holds in $L_{\chi}^{2}$. As a consequence, the following orthogonal decomposition holds,

$$
H_{\chi}^{2}=\mathbb{C} \oplus H_{\chi}^{2,1} \oplus H_{\chi}^{2,2} \oplus \cdots
$$

Proof. The orthogonal property $\varepsilon_{j}^{\mu} \perp \varepsilon_{\imath}^{\lambda}$ with $|\mu| \neq|\lambda|$ for any $\imath \in \mathbb{N}_{*}^{\ell(\lambda)}$ and $\jmath \in \mathbb{N}_{*}^{\ell(\mu)}$ follows from $(2.8)$, since

$$
\begin{aligned}
\int_{\mathfrak{U}} \varepsilon_{\jmath}^{\mu} \bar{\varepsilon}_{\imath}^{\lambda} d \chi & =\int_{\mathfrak{U}} \varepsilon_{\jmath}^{\mu}(\exp (\dot{\mathrm{i}} \vartheta) u) \bar{\varepsilon}_{\imath}^{\lambda}(\exp (\dot{\mathrm{i}} \vartheta) u) d \chi(u) \\
& =\frac{1}{2 \pi} \int_{\mathfrak{U}} \varepsilon_{\jmath}^{\mu} \bar{\varepsilon}_{\imath}^{\lambda} d \chi \int_{-\pi}^{\pi} \exp (\dot{\mathfrak{i}}(|\mu|-|\lambda|) \vartheta) d \vartheta=0
\end{aligned}
$$

for all $\lambda \in \mathbb{Y}$ and $\mu \in \mathbb{Y} \backslash\{\emptyset\}$. This yields $H_{\chi}^{2,|\mu|} \perp H_{\chi}^{2,|\lambda|}$ in the space $L_{\chi}^{2}$.

\section{Reproducing Kernels}

Let us construct the reproducing kernel of $H_{\chi}^{2}$. We refer to [19] for the basic definitions and properties of reproducing kernels.

Lemma 4.1. For every $u, v \in \mathfrak{U}$ there exists a $q \in \mathbb{N}$ such that the reproducing kernel of the subspace $H_{\chi}^{2, n}$ in $L_{\chi}^{2}$ has the form

$$
\begin{aligned}
\mathfrak{h}_{n}(v, u) & =\sum_{m \leq q}\left(\begin{array}{c}
n+m-1 \\
n
\end{array}\right)\langle\zeta(v) \mid \zeta(u)\rangle^{n} \\
& =\sum_{(\lambda, \imath) \in \mathbb{Y}_{n} \times \mathbb{N}_{*}^{\ell(\lambda)}} \frac{\varepsilon_{\imath}^{\lambda}(v) \bar{\varepsilon}_{\imath}^{\lambda}(u)}{\left\|\varepsilon_{\imath}^{\lambda}\right\|_{L_{\chi}^{2}}^{2}}, \quad u, v \in \mathfrak{U} .
\end{aligned}
$$

Proof. Note that $\mathfrak{h}_{0} \equiv 1$. From (2.3) it follows that for each stabilized sequence $u \in \mathfrak{U}$ there exists $u_{m} \in U(m)$ with a certain $m=m(u)$ such that $u=\rho\left(u_{m}\right)$. So, the element $\zeta(u)=\rho^{-1}(u) \mathfrak{e}_{1}$ is located on the $m$-dimensional sphere $\mathrm{S}(m)$. It means that its Fourier series $\zeta(u)=\sum \mathfrak{e}_{k} \varepsilon_{k}(u)$ has $m(u)$ terms. The tensor multinomial theorem yields the Fourier decomposition

$$
[\zeta(u)]^{\otimes n}=\left(\sum \mathfrak{e}_{k} \varepsilon_{k}(u)\right)^{\otimes n}=\sum_{(\lambda, \imath) \in \mathbb{Y}_{n} \times \mathbb{N}_{*}^{\ell(\lambda)}} \frac{n !}{\lambda !} \mathfrak{e}_{\imath}^{\odot \lambda} \varepsilon_{\imath}^{\lambda}(u)
$$


in the space $\mathrm{E}^{\odot n}$. Using the formula (3.2), we obtain

$$
\begin{aligned}
\langle\zeta(v) \mid \zeta(u)\rangle^{n} & =\left\langle[\zeta(v)]^{\otimes n} \mid[\zeta(u)]^{\otimes n}\right\rangle \\
& =\sum_{(\lambda, \imath) \in \mathbb{Y}_{n} \times \mathbb{N}_{*}^{\ell(\lambda)}}\left(\frac{n !}{\lambda !}\right)^{2}\left\langle\mathfrak{e}_{\imath}^{\odot \lambda} \mid \mathfrak{e}_{\imath}^{\odot \lambda}\right\rangle \varepsilon_{\imath}^{\lambda}(v) \bar{\varepsilon}_{\imath}^{\lambda}(u) \\
& =\sum_{(\lambda, \imath) \in \mathbb{Y}_{n} \times \mathbb{N}_{*}^{\ell(\lambda)}} \frac{\varepsilon_{\imath}^{\lambda}(v) \bar{\varepsilon}_{\imath}^{\lambda}(u)}{\left\|\mathfrak{e}_{\imath}^{\odot \lambda}\right\|^{2}}
\end{aligned}
$$

where $\langle\zeta(v) \mid \zeta(u)\rangle$ is decomposed into $q=\min \{m(u), m(v)\}$ summands in virtue of orthogonality. Multiplying both sides by $\left(\begin{array}{c}n+m-1 \\ n\end{array}\right)$ and summing over all $m \leq q$, we get (4.1). It follows that $\int_{\mathfrak{U}} \mathfrak{h}_{n}(v, u) \varepsilon_{\imath}^{\lambda}(u) d \chi(u)=\varepsilon_{\imath}^{\lambda}(v)$ for each $v \in \mathfrak{U}$. Via Theorem 3.1 the system $\varepsilon^{\mathbb{Y}_{n}}$ of functions $\varepsilon_{\imath}^{\lambda}$ forms an orthogonal basis in $H_{\chi}^{2, n}$. So, the integral operator

$$
\int_{\mathfrak{U}} \mathfrak{h}_{n}(v, u) \psi_{\zeta}(u) d \chi(u)=\psi_{\zeta}(v), \quad \psi_{\zeta} \in H_{\chi}^{2, n}
$$

acts identically on $H_{\chi}^{2, n}$. Thus, the kernel (4.1) is reproducing in $H_{\chi}^{2, n}$.

Let us consider the complex-valued kernel

$$
\mathfrak{h}(z ; v, u)=\prod_{m \leq \min \{m(u), m(v)\}}[1-z\langle\zeta(v) \mid \zeta(u)\rangle]^{-m}, \quad u, v \in \mathfrak{U}, \quad|z|<1
$$

where $m(u)$ is the number of terms in the Fourier series $\zeta(u)=\sum \mathfrak{e}_{k} \varepsilon_{k}(u)$.

Theorem 4.2. The expansion $\mathfrak{h}(z ; v, u)=\sum z^{n} \mathfrak{h}_{n}(v, u)$ holds for any $u, v \in \mathfrak{U}$ and $|z|<1$. The kernel $\mathfrak{h}(1 ; v, u)=\sum \mathfrak{h}_{n}(v, u)$ is reproducing in $H_{\chi}^{2}$ in the sense that

$$
\int_{\mathfrak{U}} \mathfrak{h}(1 ; v, u) f(u) d \chi(u)=f(v), \quad f \in H_{\chi}^{2}, \quad v \in \mathfrak{U} .
$$

Proof. Let $q=\min \{m(u), m(v)\}$ and $m \leq q$. As is well known [18, 1.4.10],

$$
[1-z\langle\zeta(v) \mid \zeta(u)\rangle]^{-m}=\sum_{n \in \mathbb{Z}_{+}}\left(\begin{array}{c}
n+m-1 \\
n
\end{array}\right)\langle z \zeta(v) \mid \zeta(u)\rangle^{n}
$$

for all $|z|<1$. By the Vandermonde identity, we have

$$
\begin{aligned}
\left(\begin{array}{c}
n+m-1 \\
n
\end{array}\right)\langle z \zeta(v) \mid \zeta(u)\rangle^{n} & =\left(\begin{array}{c}
r+k+p+l-2 \\
r+k
\end{array}\right)\langle z \zeta(v) \mid \zeta(u)\rangle^{r+k} \\
& =\sum_{r=0}^{n}\left(\begin{array}{c}
r+p-1 \\
r
\end{array}\right)\left(\begin{array}{c}
n-r+l-1 \\
n-r
\end{array}\right)\langle z \zeta(v) \mid \zeta(u)\rangle^{r+k}
\end{aligned}
$$


for all $n=r+k$ and $m=p+l-1$. Applying recursively this identity to the series (4.4) with any $m \leq q$ and using Lemma 4.1, we obtain

$$
\begin{aligned}
\mathfrak{h}(z ; v, u) & =\prod_{m \leq q} \sum_{n \in \mathbb{Z}_{+}}\left(\begin{array}{c}
n+m-1 \\
n
\end{array}\right)\langle z \zeta(v) \mid \zeta(u)\rangle^{n} \\
& =\sum_{n \in \mathbb{Z}_{+}} z^{n} \sum_{(\lambda, \imath) \in \mathbb{Y}_{n} \times \mathbb{N}_{*}^{\ell(\lambda)}} \frac{\varepsilon_{l}^{\lambda}(v) \bar{\varepsilon}_{\imath}^{\lambda}(u)}{\left\|\varepsilon_{\imath}^{\lambda}\right\|_{L_{\chi}^{2}}^{2}}=\sum_{n \in \mathbb{Z}_{+}} z^{n} \mathfrak{h}_{n}(v, u) .
\end{aligned}
$$

Hence, the required expansion holds. By (3.12) we have $f=\sum_{n} f_{n}$ for any $f \in H_{\chi}^{2}$ where $f_{n} \in H_{\chi}^{2, n}$ is the orthogonal projection of $f$. Observing that $\mathfrak{h}_{k}(z ; \cdot, u) \perp f_{n}(\cdot)$ with $n \neq k$ holds in $L_{\chi}^{2}$, we obtain

$$
\int_{\mathfrak{U}} \mathfrak{h}(1 ; v, u) f(u) d \chi(u)=\sum \int_{\mathfrak{U}} \mathfrak{h}_{n}(v, u) f_{n}(v) d \chi(u)=\sum f_{n}(v)=f(v)
$$

for all $v \in \mathfrak{U}$ and $f \in H_{\chi}^{2}$. Hence, (4.3) is valid.

\section{The Hilbert-Schmidt Analyticity}

Recall (see e.g. [7]) that a function $f$ on an open domain in a Banach space is said to be analytic if it is Gâteaux analytic and norm continuous. Similarly to $[6,15]$, we say that $f$ is Hilbert-Schmidt analytic if its Taylor coefficients are Hilbert-Schmidt polynomials. Now we describe a space $H^{2}$ of Hilbert-Schmidt analytic complex functions on the open ball $\mathrm{B}$.

The symmetric Fock space is defined to be the orthogonal sum

$$
\Gamma=\bigoplus_{n \in \mathbb{Z}_{+}} \mathrm{E}^{\odot n}, \quad\langle\psi \mid \phi\rangle=\sum_{n \in \mathbb{Z}_{+}}\left\langle\psi_{n} \mid \phi_{n}\right\rangle
$$

for all elements $\psi=\bigoplus_{n} \psi_{n}, \phi=\bigoplus_{n} \phi_{n} \in \Gamma$ with $\psi_{n}, \phi_{n} \in \mathrm{E}^{\odot n}$. The subset $\left\{x^{\otimes n}: x \in \mathrm{B}\right\}$ is total in $\mathrm{E} \odot n$ by virtue of (3.3). This provides the total property of the subsets $\left\{(1-x)^{-\otimes 1}: x \in \mathrm{B}\right\}$ in $\Gamma$ where we denote

$$
(1-x)^{-\otimes 1}:=\sum x^{\otimes n}, \quad x^{\otimes 0}=1 .
$$

The $\Gamma$-valued function $(1-x)^{-\otimes 1}$ in the variable $x \in \mathrm{B}$ is analytic, since

$$
\left\|(1-x)^{-\otimes 1}\right\|^{2}=\sum\|x\|^{2 n}=\left(1-\|x\|^{2}\right)^{-1}<\infty .
$$

Let us define the Hilbert space of analytic complex functions in the variable $x \in \mathrm{B}$, associated with the Fock space $\Gamma$, as follows

$$
H^{2}=\left\{\psi^{*}(x)=\left\langle(1-x)^{-\otimes 1} \mid \psi\right\rangle: \psi \in \Gamma\right\}, \quad\left\|\psi^{*}\right\|_{H^{2}}:=\|\psi\|
$$

for all $x \in \mathrm{B}$. This description is correct, because each function $\psi^{*}$ in the variable $x \in \mathrm{B}$ is analytic by virtue of [9, Prop. 2.4.2], as a composition of the analytic $\Gamma$-valued function $(1-x)^{-\otimes 1}$ in the variable $x \in \mathrm{B}$ and the linear functional $\langle\cdot \mid \psi\rangle$ on $\Gamma$. 
Similarly, we define the closed subspace in $H^{2}$ of $n$-homogenous HilbertSchmidt polynomials $\psi_{n}^{*}$ in the variable $x \in \mathrm{E}$ as

$$
H_{n}^{2}=\left\{\psi_{n}^{*}(x)=\left\langle x^{\otimes n} \mid \psi_{n}\right\rangle: \psi_{n} \in \mathrm{E}^{\odot n}\right\} .
$$

Differentiating at zero any function $\psi^{*}=\bigoplus \psi_{n}^{*} \in H^{2}$ with $\psi_{n}^{*} \in H_{n}^{2}$, we obtain that its Taylor coefficients at zero $(n !)^{-1} d_{0}^{n} \psi^{*}=\psi_{n}^{*}$ are Hilbert-Schmidt polynomials. Hence, every function from $H^{2}$ is Hilbert-Schmidt analytic. Clearly, the following orthogonal decomposition holds,

$$
H^{2}=\mathbb{C} \oplus H_{1}^{2} \oplus H_{2}^{2} \oplus \cdots .
$$

One can show that $\left(H_{n}^{2}\right)_{n}$ is a coherent sequence of polynomial ideals over $\mathrm{E}$ in the meaning of [4, Def. 1.1].

For each pair $(\lambda, \imath) \in \mathbb{Y}_{n} \times \mathbb{N}_{*}^{\ell(\lambda)}$, we can uniquely assign the HilbertSchmidt $n$-homogenous polynomial

$$
\hat{x}_{\imath}^{\lambda}:=\left\langle x^{\otimes n} \mid \mathfrak{e}_{\imath}^{\odot \lambda}\right\rangle, \quad x \in \mathrm{E},
$$

defined via the Fourier coefficients $\hat{x}_{k}:=\mathfrak{e}_{k}^{*}(x)=\left\langle x \mid \mathfrak{e}_{k}\right\rangle$ of an element $x \in \mathrm{E}$. Taking into account (3.2), the tensor multinomial theorem yields the following orthogonal decompositions with respect to the basis $\mathfrak{e}^{\odot \mathbb{Y}}$ in $\Gamma$,

$$
(1-x)^{-\otimes 1}=\sum_{(\lambda, \imath) \in \mathbb{Y} \times \mathbb{N}_{*}^{\ell(\lambda)}} \frac{\hat{x}_{\imath}^{\lambda} \mathfrak{e}_{\imath}^{\odot \lambda}}{\left\|\mathfrak{e}_{\imath}^{\odot \lambda}\right\|^{2}}, \quad x \in \mathrm{B} .
$$

Hence, any function $\psi^{*} \in H^{2}$ has the orthogonal expansion

$$
\psi^{*}(x)=\left\langle(1-x)^{-\otimes 1} \mid \psi\right\rangle=\sum_{(\lambda, \imath) \in \mathbb{Y} \times \mathbb{N}_{*}^{\ell(\lambda)}} \hat{\psi}_{(\lambda, \imath)} \hat{x}_{\imath}^{\lambda}, \quad x \in \mathrm{B}
$$

where $\hat{\psi}_{(\lambda, \imath)}:=\left\langle\mathfrak{e}_{\imath}^{\odot \lambda} \mid \psi\right\rangle\left\|\mathfrak{e}_{\imath}^{\odot \lambda}\right\|^{-2}$ are the Fourier coefficients of $\psi \in \Gamma$ with respect to the basis $\mathfrak{e}^{\odot \mathbb{Y}}$ and, moreover, $\left\|\psi^{*}\right\|_{H^{2}}^{2}=\sum_{(\lambda, \imath)}\left|\left\langle\mathfrak{e}_{\imath}^{\odot \lambda} \mid \psi\right\rangle\right|^{2}\left\|\mathfrak{e}_{\imath}^{\odot \lambda}\right\|^{-2}$. Thus, $\left\|\psi^{*}\right\|_{H^{2}}$ is a Hilbert-Schmidt type norm on $H^{2}$.

\section{Integral Formulas}

The one-to-one correspondence $\mathfrak{e}_{i}^{\odot \lambda} \leftrightarrow \varepsilon_{\imath}^{\lambda}$ allows us to construct an antilinear isometric isomorphism $\mathcal{J}: \Gamma \longrightarrow H_{\chi}^{2}$ and its adjoint $\mathcal{J}^{*}: H_{\chi}^{2} \longrightarrow \Gamma$ by the following change of orthonormal bases

$$
\mathcal{J}: \Gamma \ni \mathfrak{e}_{\imath}^{\odot \lambda}\left\|\mathfrak{e}_{\imath}^{\odot \lambda}\right\|^{-1} \longmapsto \varepsilon_{\imath}^{\lambda}\left\|\varepsilon_{\imath}^{\lambda}\right\|_{L_{\chi}^{2}}^{-1} \in H_{\chi}^{2}, \quad \lambda \in \mathbb{Y}, \quad \imath \in \mathbb{N}_{*}^{\ell(\lambda)} .
$$

Clearly, $\mathcal{J}^{*}: \varepsilon_{\imath}^{\lambda}\left\|\varepsilon_{\imath}^{\lambda}\right\|_{L_{\chi}^{2}}^{-1} \longmapsto \mathfrak{e}_{\imath}^{\odot \lambda}\left\|\mathfrak{e}_{\imath}^{\odot \lambda}\right\|^{-1}$, because $\left\langle\mathcal{\partial} \mathfrak{e}_{\imath}^{\odot \lambda} \mid f\right\rangle_{L_{\chi}^{2}}=\left\langle\mathfrak{e}_{\imath}^{\odot \lambda} \mid \mathcal{J}^{*} f\right\rangle$ for any $f \in H_{\chi}^{2}$. Using Theorem 3.2, for any element $\psi \in \Gamma$ with the Fourier coefficients $\hat{\psi}_{(\lambda, \imath)}=\left\langle\mathfrak{e}_{\imath}^{\odot \lambda} \mid \psi\right\rangle\left\|\mathfrak{e}_{\imath}^{\odot \lambda}\right\|^{-2}$, we obtain 


$$
\partial \psi=\sum_{(\lambda, \imath) \in \mathbb{Y} \times \mathbb{N}_{*}^{\ell(\lambda)}} \hat{\psi}_{(\lambda, \imath)} \frac{\left\|\mathfrak{e}_{\imath}^{\odot \lambda}\right\|^{2}}{\left\|\varepsilon_{\imath}^{\lambda}\right\|_{L_{\chi}^{2}}^{2}} \varepsilon_{\imath}^{\lambda} \quad \text { where } \quad \frac{\left\|\mathfrak{e}_{\imath}^{\odot \lambda}\right\|^{2}}{\left\|\varepsilon_{\imath}^{\lambda}\right\|_{L_{\chi}^{2}}^{2}}=\frac{(\ell(\lambda)-1+|\lambda|) !}{(\ell(\lambda)-1) !|\lambda| !} .
$$

In particular, $\mathcal{J} x=\sum \hat{x}_{k} \varepsilon_{k}$ for any elements $x \in \mathrm{E}$ with the Fourier coefficients $\hat{x}_{k}=\left\langle x \mid \mathfrak{e}_{k}\right\rangle$. Moreover, $\|\mathcal{\partial} x\|_{L_{\chi}^{2}}^{2}=\sum\left\|\hat{x}_{k}\right\|^{2}=\|x\|^{2}$.

In what follows, we assign to each $x \in \mathrm{E}$ the $L_{\chi}^{2}$-valued function

$$
x_{\mathfrak{J}}: \mathfrak{U} \ni u \longmapsto(\mathcal{J} x)(u) .
$$

Lemma 6.1. The function $\mathcal{J}(1-x)^{-\otimes 1}=\left(1-x_{\mathfrak{J}}\right)^{-1}$ in the variable $u \in \mathfrak{U}$ takes values in $L_{\chi}^{2}$ for all $x \in \mathrm{B}$.

Proof. Applying $\mathcal{J}$ to the decompositions (3.1) and (5.3), we obtain

$$
\begin{aligned}
\mathcal{J}(1-x)^{-\otimes 1} & =\sum_{(\lambda, \imath) \in \mathbb{Y} \times \mathbb{N}_{*}^{\ell(\lambda)}} \frac{\hat{x}_{\imath}^{\lambda} \varepsilon_{\imath}^{\lambda}}{\left\|\mathfrak{e}_{\imath}^{\odot \lambda}\right\|^{2}} \\
& =\sum_{n \in \mathbb{Z}_{+}}\left(\sum_{k \in \mathbb{N}} \hat{x}_{k} \varepsilon_{k}\right)^{n}=\left(1-x_{\mathfrak{J}}\right)^{-1}
\end{aligned}
$$

where the following orthogonal series with a fixed $n \in \mathbb{N}$,

$$
x_{\mathfrak{J}}^{n}=\left(\sum_{k \in \mathbb{N}} \hat{x}_{k} \varepsilon_{k}\right)^{n}=\sum_{(\lambda, \imath) \in \mathbb{Y}_{n} \times \mathbb{N}_{*}^{\ell(\lambda)}} \frac{\hat{x}_{\imath}^{\lambda} \varepsilon_{\imath}^{\lambda}}{\left\|\mathfrak{e}_{\imath}^{\odot \lambda}\right\|^{2}},
$$

is convergent in $L_{\chi}^{2}$. Moreover, taking into account the orthogonality, we get

$$
\begin{aligned}
\left\|\left(1-x_{\mathcal{J}}\right)^{-1}\right\|_{L_{\chi}^{2}}^{2} & =\sum_{n \in \mathbb{Z}_{+}} \sum_{(\lambda, \imath) \in \mathbb{Y}_{n} \times \mathbb{N}_{*}^{\ell(\lambda)}} \frac{\left|\hat{x}_{\imath}^{\lambda}\right|^{2}}{\left\|\mathfrak{e}_{\imath}^{\odot \lambda}\right\|^{2}} \\
& =\sum_{n \in \mathbb{Z}_{+}}\left(\sum_{k \in \mathbb{N}}\left|\hat{x}_{k}\right|^{2}\right)^{n}=\left(1-\|x\|^{2}\right)^{-1} .
\end{aligned}
$$

Hence, the function $\left(1-x_{\mathfrak{\jmath}}\right)^{-1}$ with $x \in \mathrm{B}$ takes values in $L_{\chi}^{2}$.

Let $f=\sum_{n} f_{n} \in H_{\chi}^{2}$ with $f_{n} \in H_{\chi}^{2, n}$. Then $\mathfrak{g}^{*} f \in \Gamma$ and $\mathrm{J}^{*} f_{n} \in \mathrm{E} \odot n$. Briefly denote $\tilde{f}:=\left(\mathcal{J}^{*} f\right)^{*} \in H_{n}^{2}$ and $\tilde{f}_{n}:=\left(\mathcal{J}^{*} f_{n}\right)^{*} \in H^{2}$. Thus,

$$
\begin{aligned}
\tilde{f}(x) & =\left\langle(1-x)^{-\otimes 1} \mid \mathcal{J}^{*} f\right\rangle, \quad x \in \mathrm{B}, \\
\tilde{f}_{n}(x) & =\left\langle x^{\otimes n} \mid \mathcal{J}^{*} f_{n}\right\rangle, \quad x \in \mathrm{E} .
\end{aligned}
$$

Theorem 6.2. Each Hilbert-Schmidt analytic function $\tilde{f} \in H^{2}$ has the integral representation

$$
\tilde{f}(x)=\int_{\mathfrak{U}} \frac{f d \chi}{1-x_{\mathfrak{\jmath}}}, \quad x \in \mathrm{B}
$$

and its Taylor coefficients at zero have the form

$$
\frac{d_{0}^{n} \tilde{f}(x)}{n !}=\int_{\mathfrak{U}} x_{\mathfrak{J}}^{n} f_{n} d \chi, \quad x \in \mathrm{E} .
$$


The mapping $f \longmapsto \tilde{f}$ produces a linear isometry $H_{\chi}^{2} \simeq H^{2}$.

Proof. Consider the Fourier decomposition of $f$ with respect to the basis $\varepsilon^{\mathbb{Y}}$ and its $\mathcal{J}^{*}$-image, respectively

$$
f=\sum_{(\lambda, \imath) \in \mathbb{Y} \times \mathbb{N}_{*}^{e}(\lambda)} \hat{f}_{(\lambda, \imath)} \varepsilon_{\imath}^{\lambda}, \quad g^{*} f=\sum_{(\lambda, \imath) \in \mathbb{Y} \times \mathbb{N}_{*}^{\ell(\lambda)}} \overline{\hat{f}}_{(\lambda, \imath)} \frac{\left\|\varepsilon_{\imath}^{\lambda}\right\|_{L_{\chi}^{2}}^{2}}{\left\|\mathfrak{e}_{\imath}^{\odot \lambda}\right\|^{2}} \mathfrak{e}_{\imath}^{\odot \lambda}
$$

where $\hat{f}_{(\lambda, \imath)}=\left\|\varepsilon_{\imath}^{\lambda}\right\|_{L_{\chi}^{2}}^{-2} \int_{\mathfrak{U}} f \bar{\varepsilon}_{\imath}^{\lambda} d \chi$. Substituting $\hat{f}_{(\lambda, \imath)}$ to $\tilde{f}=\left(\mathcal{J}^{*} f\right)^{*}$ and using the orthogonal property and the relations (5.3) and (6.1), we obtain

$$
\begin{aligned}
\tilde{f}(x) & =\sum_{(\lambda, \imath) \in \mathbb{Y} \times \mathbb{N}_{*}^{\ell(\lambda)}} \frac{\hat{f}_{(\lambda, \imath)} \hat{x}_{l}^{\lambda}\left\langle\mathfrak{e}_{i}^{\odot \lambda} \mid \mathfrak{e}_{i}^{\odot \lambda}\right\rangle\left\|\varepsilon_{l}^{\lambda}\right\|_{L_{\chi}^{2}}^{2}}{\left\|\mathfrak{e}_{\imath}^{\odot \lambda}\right\|^{4}} \\
& =\int_{\mathfrak{U}} \sum_{(\lambda, \imath) \in \mathbb{Y} \times \mathbb{N}_{*}^{\ell(\lambda)}} \frac{\hat{x}_{\imath}^{\lambda} \varepsilon_{l}^{\lambda}}{\left\|\mathfrak{e}_{\imath}^{\odot \lambda}\right\|^{2}} f d \chi=\int_{\mathfrak{U}} \frac{f d \chi}{1-x_{\mathfrak{d}}} .
\end{aligned}
$$

Hence, (6.3) holds. Using (6.2), we similarly obtain

$$
\tilde{f}_{n}(x)=\left\langle x^{\otimes n} \mid \partial^{*} f_{n}\right\rangle=\int_{\mathfrak{U}} x_{\mathfrak{J}}^{n} f_{n} d \chi .
$$

Taking into account (6.5) and the orthogonal decomposition (3.12), we get

$$
\tilde{f}(\alpha x)=\left\langle(1-\alpha x)^{-\otimes 1} \mid \mathcal{J}^{*} f\right\rangle=\sum \alpha^{n} \int_{\mathfrak{U}} x_{\mathfrak{J}}^{n} f_{n} d \chi, \quad|\alpha| \leq 1 .
$$

Note that $\tilde{f}(\alpha x)$ is analytic in $\alpha$ for all $x \in \mathrm{B}$. Differentiating $\tilde{f}(\alpha x)$ at $\alpha=0$ and using the $n$-homogeneity of derivatives, we obtain

$$
\left.\frac{d^{n}}{d \alpha^{n}} \sum \alpha^{n} \int_{\mathfrak{U}} x_{\mathfrak{J}}^{n} f_{n} d \chi\right|_{\alpha=0}=n ! \int_{\mathfrak{U}} x_{\mathfrak{J}}^{n} f_{n} d \chi .
$$

Hence, the functions (6.4) coincide with the Taylor coefficients at zero of $\tilde{f}$.

Finally, since the image of $\varepsilon^{\mathbb{Y}}$ under $\mathcal{J}^{*}$ coincides with $\mathfrak{e}^{\odot \mathbb{Y}}$, the mapping $H_{\chi}^{2} \ni f \longmapsto \tilde{f} \in H^{2}$ is an isometry.

\section{Radial Boundary Values}

Using (6.3), for each $f=\sum_{n} f_{n} \in H_{\chi}^{2}$ with $f_{n} \in H_{\chi}^{2, n}$ we can rewrite (6.6) as

$$
\tilde{f}(r x)=\left\langle(1-r x)^{-\otimes 1} \mid \partial^{*} f\right\rangle=\int_{\mathfrak{U}} \frac{f d \chi}{1-r x_{\mathfrak{J}}}, \quad x \in \mathrm{K}, \quad r \in[0,1)
$$

where $\mathrm{K}=\{x \in \mathrm{E}:\|x\| \leq 1\}$.

Theorem 7.1. The integral transform $\mathcal{C}_{r}: f \longmapsto \mathcal{C}_{r}[f]$, defined as

$$
\mathcal{C}_{r}[f](x):=\int_{\mathfrak{U}} \frac{f d \chi}{1-r x_{\mathfrak{J}}}, \quad x \in \mathrm{K}, \quad r \in[0,1),
$$


belongs to the space of bounded linear operators $\mathscr{L}\left(H_{\chi}^{2}, H^{2}\right)$. The radial boundary values of $\mathcal{C}_{r}[f] \in H^{2}$ are equal to $\tilde{f} \in H^{2}$ in the following sense:

$$
\lim _{r \nearrow 1}\left\|\mathfrak{C}_{r}[f]-\tilde{f}\right\|_{H^{2}}=0 .
$$

Moreover, the following equality holds,

$$
\|\tilde{f}\|_{H^{2}}^{2}=\sup _{r \in[0,1)}\left\|\mathfrak{e}_{r}[f]\right\|_{H^{2}}^{2} .
$$

Proof. Theorem 6.2 and (7.1) imply the equality $\mathcal{C}_{r}[f]=\sum r^{n} \tilde{f}_{n}$ for any $r \in[0,1)$. By $(5.2)$, we have $\tilde{f}_{k} \perp \tilde{f}_{n}$ as $n \neq k$ in $H^{2}$. It follows that

$$
\left\|\mathcal{C}_{r}[f]\right\|_{H^{2}}^{2}=\left\|\sum r^{n} \tilde{f}_{n}\right\|_{H^{2}}^{2}=\sum r^{2 n}\left\|\tilde{f}_{n}\right\|_{H^{2}}^{2}=\sum r^{2 n}\left\|f_{n}\right\|_{L_{\chi}^{2}}^{2},
$$

since $\mathcal{J}^{*}$ acts isometrically from $H_{\chi}^{2, n}$ onto the space $\mathrm{E}^{\odot n}$ which is antilinear isometric to $H_{n}^{2}$ by definition. Similarly, we obtain that

$$
\left\|\mathcal{C}_{r}[f]-\tilde{f}\right\|_{H^{2}}^{2}=\sum\left(r^{2 n}-1\right)\left\|f_{n}\right\|_{L_{\chi}^{2}}^{2} \longrightarrow 0, \quad r \rightarrow 1 .
$$

Moreover, the Cauchy-Schwarz inequality implies that

$$
\left\|\mathcal{C}_{r}[f]\right\|_{H^{2}}^{2} \leq \frac{1}{\left(1-r^{2}\right)^{1 / 2}}\left(\sum\left\|f_{n}\right\|_{L_{\chi}^{2}}^{2}\right)^{1 / 2}=\frac{\|f\|_{L_{\chi}^{2}}}{\left(1-r^{2}\right)^{1 / 2}}
$$

for all $f \in H_{\chi}^{2}$. Hence, the operator $\mathcal{C}_{r}$ belongs to $\mathscr{L}\left(H_{\chi}^{2}, H^{2}\right)$ for all $r \in[0,1)$.

Finally, the equalities

$$
\sup _{r \in[0,1)}\left\|\mathfrak{C}_{r}[f]\right\|_{H^{2}}^{2}=\sup _{r \in[0,1)} \sum r^{2 n}\left\|\tilde{f}_{n}\right\|_{H^{2}}^{2}=\sum\left\|\tilde{f}_{n}\right\|_{H^{2}}^{2}=\|\tilde{f}\|_{H^{2}}^{2}
$$

give the required formula (7.3).

\section{Acknowledgements}

I am grateful to the referees for their comments and valuable suggestions which greatly improved this article.

Open Access. This article is distributed under the terms of the Creative Commons Attribution 4.0 International License (http://creativecommons.org/licenses/ by/4.0/), which permits unrestricted use, distribution, and reproduction in any medium, provided you give appropriate credit to the original author(s) and the source, provide a link to the Creative Commons license, and indicate if changes were made. 


\section{References}

[1] Berezanski, Yu.M., Kondratiev, Yu.G.: Spectral Methods in Infinite-Dimensional Analysis. Springer, Berlin (1995)

[2] Borodin, A., Olshanski, G.: Harmonic analysis on the infinite-dimensional unitary group and determinantal point processes. Ann. Math. 161, 1319-1422 (2005)

[3] Borodin, A: Determinantal point processes. In: Akemann, G., Baik, J., Di Francesco, P. (eds.) Oxford Handbook of Random Matrix Theory, Oxford Univ. Press, Oxford (2011)

[4] Carando, D., Dimant, V., Muro, S.: Coherent sequences of polynomial ideals on Banach spaces. Math. Nachr. 282(8), 1111-1133 (2009)

[5] Cole, B., Gamelin, T.W.: Representing measures and Hardy spaces for the infinite polydisk algebra. Proc. Lond. Math. Soc. 53, 112-142 (1986)

[6] Dwyer III, T.A.W.: Partial differential equations in Fischer-Fock spaces for the Hilbert-Schmidt holomorphy type. Bull. Am. Math. Soc. 77(5), 725-739 (1971)

[7] Gamelin, T.W: Analytic functions on Banach spaces. In: Gauthier, P.M., Sabidussi, G. (eds.) Complex Function Theory, pp. 87-223. Kluwer, Dordrecht (1994)

[8] Floret, K.: Natural norms on symmetric tensor products of normed spaces. Note di Matematica 17, 153-188 (1997)

[9] Hervé, M.: Analyticity in Infiite Dimensional Spaces, de Gruyter Stud. in Math., vol. 10. Walter de Gruyter, Berlin (1989)

[10] Hewitt, E., Ross, K.A.: Abstract Harmonic Analysis, vol. 2. Springer, Berlin (1994)

[11] Lopushansky, O.: Hardy type space associated with an infinite-dimensional unitary matrix group. Abst. Appl. An. ID 810735, 1-7 (2013)

[12] Neretin, Yu.A.: Hua type integrals over unitary groups and over projective limits of unitary groups. Duke Math. J. 114(2), 239-266 (2002)

[13] Olshanski, G.: The problem of harmonic analysis on the infinite-dimensional unitary group. J. Funct. Anal. 205, 464-524 (2003)

[14] Neeb, K.H., Ørted, B.: Hardy spaces in an infinite dimensional setting. In: Doebner, H.D. (ed.) Lie Theory and Its Applications in Physics, pp. 3-27. Word Sci. Publ., Singapore (1998)

[15] Petersson, P.: Hypercyclic convolution operators on entire functions of HilbertSchmidt holomorphy type. Ann. Math. Blaise Pascal 8(2), 107-114 (2001)

[16] Pickrell, D.: Measures on infinite-dimensional Grassmann manifolds. J. Funct. Anal. 70, 323-356 (1987)

[17] Pinasco, D., Zalduendo, I.: Integral representations of holomorphic functions on Banach spaces. J. Math. Anal. Appl. 308, 159-174 (2005)

[18] Rudin, W.: Function Theory in the Unit Ball of $\mathbb{C}^{n}$. Springer, Berlin (2008)

[19] Saitoh, S.: Integral Transforms, Reproducing Kernels and Their Applications. Pitman Research Notes in Math. Ser., vol. 369. Longman (1997) 
Oleh Lopushansky

Faculty of Mathematics and Natural Sciences

Rzeszów University

1 Pigonia str.

35-310 Rzeszów

Poland

e-mail: ovlopusz@ur.edu.pl

Received: February 20, 2015.

Accepted: April 1, 2016. 